\title{
ASYMMETRIC $P^{\prime} P^{\prime}$ : AN ALTERNATIVE TO $P^{\prime} d P^{\prime}$ REFLECTIONS IN THE UPPERMOST MANTLE (0 to $110 \mathrm{KM}$ )
}

\author{
BY JAMES H. WhITCOMB
}

\begin{abstract}
Precursors to $\boldsymbol{P}^{\prime} \boldsymbol{P}^{\prime}(\boldsymbol{P K P P K P})$, first interpreted as sub-surface reflections by Gutenberg in 1960 and studied in several later papers by other authors, precede the $P^{\prime} P^{\prime}$ phase by up to $200 \mathrm{sec}$. This phase, designated $P^{\prime} d P^{\prime}$ where $d$ is the depth of reflection, has unique potential for giving new details of upper-mantle structure. However, as with any newly discovered seismic phase, the uniqueness of its interpretation must be well established. Asymmetric $\boldsymbol{P}^{\prime} \boldsymbol{P}^{\prime}$ phases reflecting from surface or near-surface dipping interfaces pose a challenge to this uniqueness because of their maximum-time nature. Simplified estimates of the amplitudes of asymmetric $\boldsymbol{P}^{\prime} \boldsymbol{P}^{\prime}$ rays are made, including consideration of the relative amplitudes of core phases and the finiteness of the reflecting surfaces of dipping interfaces. These estimates lead to the conclusion that the reading of asymmetric $P^{\prime} P^{\prime}$ at a single station is likely only in the 0 - to 30 -sec range before the main symmetric $P^{\prime} P^{\prime}$ phase. However, if array beam-forming is used, this range is reduced to 0 to 10 sec. The data indicate that both $P^{\prime} d P^{\prime}$ and asymmetric $P^{\prime} P^{\prime}$ are present at up to $30 \mathrm{sec}$ lead time and array beam-forming is needed to differentiate between the two. A further effect of the maximum-time nature of $\boldsymbol{P}^{\prime} \boldsymbol{P}^{\prime}$ is that, in practice, the geographic location of the reflection point can be determined to within only a few degrees.
\end{abstract}

\section{INTRODUCTION}

Precursors up to $30 \mathrm{sec}$ before $P^{\prime} P^{\prime}(P K P P K P)$ were first noted by Gutenberg (1960) and were interpreted as sub-surface reflections. Later authors including Adams (1968), Engdahl and Flinn (1969a), Whitcomb and Anderson (1970), Whitcomb (1971), Adams (1971), and Richards (1972) have made studies of these and earlier precursors based on the same interpretation that they are reflections within the upper mantle, i.e., $P^{\prime} d P^{\prime}$ (as defined by Whitcomb and Anderson, 1970) where $d$ is the depth of reflection.

This new data type is important because it can give details of upper-mantle structure heretofore unavailable, and provides one of the few means at hand for comparison of mantle structure under different lithospheric regions such as continents and oceans. However, as with any newly discovered seismic phase, the uniqueness of its interpretation must be well established. A violation of this uniqueness was pointed out by Engdahl and Flinn (1969b), who showed that the phase $S K K K P$ for deep earthquakes occurs in the same part of the travel-time curve and could be mistaken for $P^{\prime} d P^{\prime}$. This problem is eliminated by avoiding readings at the time of $S K K K P$ at a single station, or by using the difference in $d t / d \Delta$ between $S K K K P$ and $P^{\prime} d P^{\prime}$ at an array of stations.

A much more difficult problem is posed by the maximum-time nature of $P^{\prime} P^{\prime}$ phases discussed by Jeffreys and Lapwood (1957) and later by Richards (1972) and Wright (1972). Asymmetric $P^{\prime} P^{\prime}$ phases reflecting from dipping interfaces at or near the Earth's surface would arrive earlier than the main phase for all branches except for a special path of the $A B$ branch. This paper deals with the estimation of asymmetric $P^{\prime} P^{\prime}$ amplitudes and, therefore, the likelihood that these phases be misinterpreted as $P^{\prime} d P^{\prime}$. 


\section{ASYMMETRIC REFLECTIONS OF $P^{\prime} P^{\prime}$}

$P^{\prime} P^{\prime}$ is a true maximum-time phase with the exception of the receding $A B$ branch. Specifically, if $P^{\prime} P^{\prime}$ reflects from any nonhorizontal surface of the Earth at a nonsymmetrical point, it will arrive earlier than its symmetrical counterpart. This is not true for the receding $A B$ branch reflecting on the station-epicenter great circle; the $A B$ branch is thus a "mini-max" phase (in the reverse sense of the phase $P P$ for which asymmetric reflections on the great circle arrive earlier). It will be seen, however, that asymmetric reflections off the great circle are most important and for our purposes the $A B$ branch is effectively a maximum-time phase.

For convenience of calculation, asymmetric $P^{\prime} P^{\prime}$ reflections are divided into two types as shown in Figure 1: those in the great-circle plane and those out of the great-circle plane reflecting at points on a line that is perpendicular to the great circle and passes

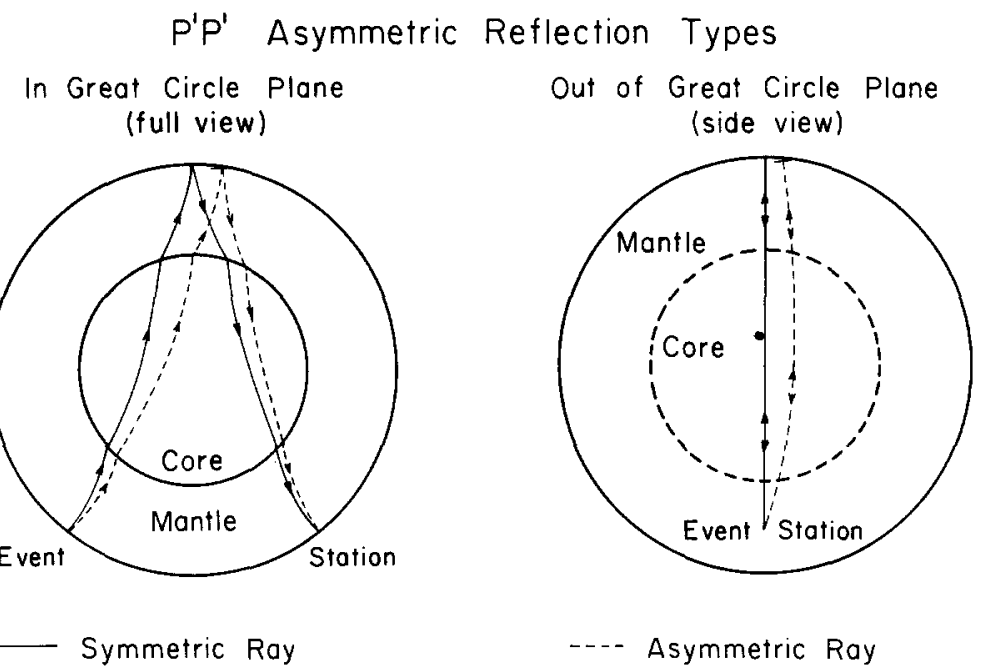

FIG. 1. $P^{\prime} P^{\prime}$ asymmetric reflection types. Symmetric rays are shown for reference.

through the symmetrical reflection point. Of course, any combination of these two types is permitted. The symmetric ray is shown in each case in Figure 1. In all of the calculations, the nonhorizontal reflector is assumed to be at or very near the Earth's surface where the largest reflection amplitudes are expected. The reflectivity coefficient of the Moho is about 0.14 (Whitcomb and Anderson, 1970). This reduces the amplitude almost to a 10 per cent level and the asymmetric reflection from this interface is probably not important. The most likely candidates for asymmetric reflectors are a nonhorizontal ocean bottom or land surface; the former's reflectivity can be as high as 0.8 and the latter's is 1.0. Therefore, no reduction in amplitude is made for asymmetric phase reflectivity coefficients because they may be equal to that of the symmetric phase.

Another possibility for the generation of asymmetric $P^{\prime} P^{\prime}$ phases lies in the distortions of $d t / d \Delta$ produced by inhomogeneities in the upper mantle. A good example is a cold lithospheric slab sinking into the mantle at trench areas. However, the calculations of Julian (1970, Figure 2) show that, for such a complex structure, the anisotropy scatters the rays too severely to reflect from the surface a second core phase of significant amplitude. Broader anisotropic structures in the upper mantle might be more effective in evenly 
distorting a large cone of $P^{\prime} P^{\prime}$ energy, but calculations of this effect must await a better understanding of broad lateral variations in upper-mantle structure.

\section{OBSERVED AMPLITUDES}

Figure 2 shows schematic travel-time curves of the branches of $P^{\prime} P^{\prime}$ and $P^{\prime} d P^{\prime}$. Some question still persists about the existence of the $G H$ branch at distances greater than $73^{\circ}$ but arrivals earlier than $D F$ in this range are small and are not important for this computation. The relative amplitudes (amplitudes relative to the $D F$ branch) of the $P^{\prime}$ branches have been summarized mainly from data of Shahidi (1968), Buchbinder (1971), and inferences from $P^{\prime} P^{\prime}$ data of Whitcomb (1971) and are shown in Figure 3. These ampli-

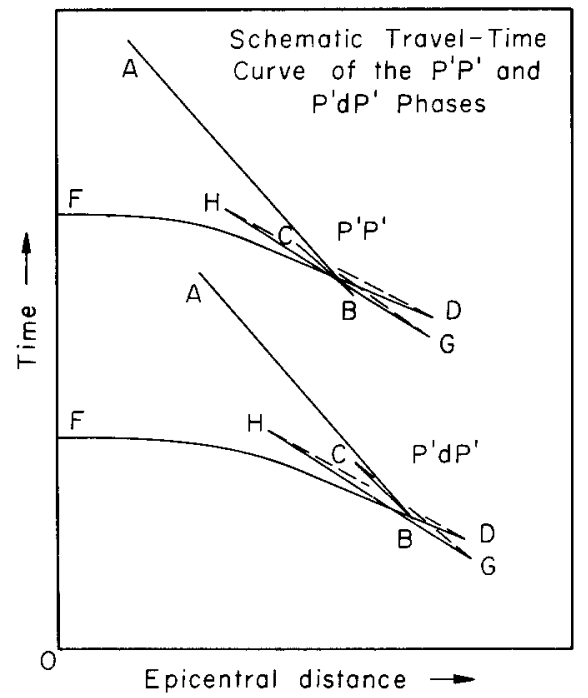

FIG. 2. Schematic travel-time curves of the branches of $P^{\prime} P^{\prime}$ and $P^{\prime} d P^{\prime}$.

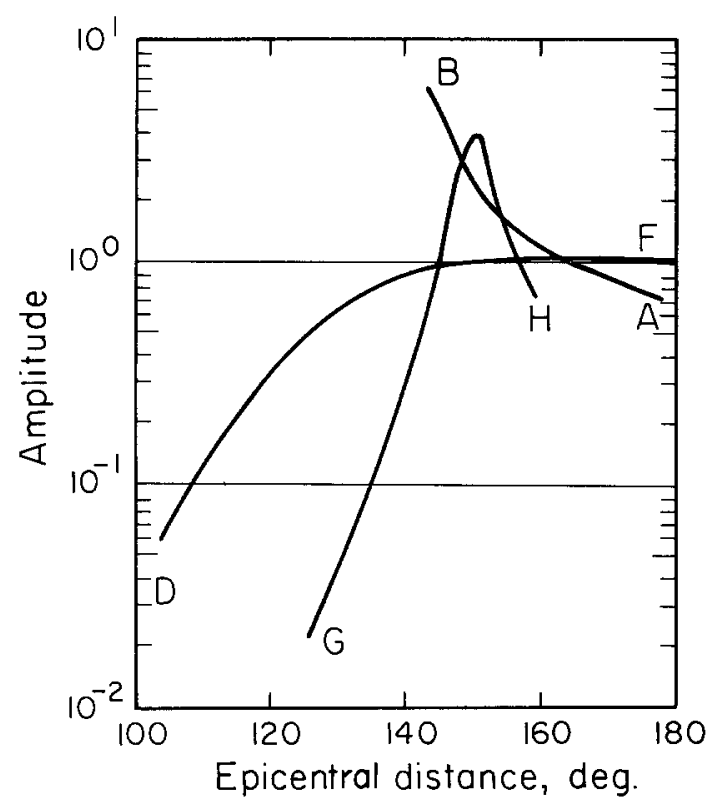

Fig. 3. Observed amplitudes of the branches of $P^{\prime}$ normalized by the $D F$ branch at $180^{\circ}$ epicentral distance. 
tude curves are approximate fits to the actual data, which, as is typical, scatter by as much as 50 per cent. However, the $P^{\prime}$ amplitudes contrast so markedly as a function of distance and the branch that useful estimates can be made of the relative amplitude of a specified ray.

The $P^{\prime} P^{\prime}$ precursor amplitudes are 30 to 50 per cent of the main $P^{\prime} P^{\prime}$ phase at lead times of 0 to $30 \mathrm{sec}$ and 10 to 20 per cent of the main phase at lead times greater than $30 \mathrm{sec}$ (Whitcomb and Anderson, 1970). We initially assume, and later justify, that only the 0 - to 30 -sec precursors are candidates for asymmetric $P^{\prime} P^{\prime}$. Therefore, we can define a likelihood criterion as follows: when the asymmetric $P^{\prime} P^{\prime}$ amplitudes fall below 10 per cent of the theoretical symmetric phase, they are too small to be identified as readings. That is, when the calculated asymmetric $P^{\prime} P^{\prime}$ amplitudes fall below one-third of the smallest actual readings in that time-window, we conclude that the readings are not asymmetric $P^{\prime} P^{\prime}$.

\section{THeORETICAL AMPLitudes OF SYMMETRIC $P^{\prime} P^{\prime}$}

Because the amplitudes of $P^{\prime} P^{\prime}$ are not as well known as those of $P^{\prime}$, we translate $P^{\prime}$ into symmetric $P^{\prime} P^{\prime}$ amplitudes for use as comparisons. From Bullen (1963, p. 127), the energy density resulting from geometrical spreading of a seismic ray at the Earth's surface can be written in the form

$$
E(\Delta)=\frac{B f}{\sin \Delta}\left|\frac{d^{2} T(\Delta)}{d \Delta^{2}}\right|
$$

where $B$ is a constant, $f$ is a function depending mainly on emergence angle, $T$ is the travel time, and $\Delta$ is epicentral distance.

Suppose now that $T_{1}(\Delta)$ corresponds to $P^{\prime}$ of the $D F$ branch for a surface focus and $T_{11}(2 \Delta)$ corresponds to $P^{\prime} P^{\prime}$ of the same branch. From symmetry we can write

$$
T_{11}(2 \Delta)=2 T_{1}(\Delta)
$$

or, defining $\Delta^{\prime}=2 \Delta,(2)$ becomes

$$
T_{11}\left(\Delta^{\prime}\right)=2 T_{1}(\Delta)
$$

Then, differentiating twice with respect to $\Delta^{\prime}$, we have

$$
\left|\frac{d^{2} T_{11}\left(\Delta^{\prime}\right)}{d\left(\Delta^{\prime}\right)^{2}}\right|=\frac{1}{2}\left|\frac{d^{2} T_{1}(\Delta)}{d \Delta^{2}}\right| \text {. }
$$

The energies for the $P^{\prime}$ and $P^{\prime} P^{\prime}$ phases, $D F$ branch, are then, from equation (1),

$$
E_{1}(\Delta)=\frac{B_{1} f_{1}}{\sin \Delta}\left|\frac{d^{2} T_{1}(\Delta)}{d \Delta^{2}}\right|
$$

and

$$
E_{11}\left(\Delta^{\prime}\right)=\frac{B_{1} f_{1}}{\sin (2 \Delta)} \frac{1}{2}\left|\frac{d^{2} T_{1}(\Delta)}{d \Delta^{2}}\right|
$$

respectively; where again $\Delta$ corresponds to the $P^{\prime}$ epicentral distance and $\Delta^{\prime}$ to the $P^{\prime} P^{\prime}$ distance for the same part of the branch.

The energy expressions (5) and (6) can be related to amplitude by (Bullen, 1963, p. 123)

$$
E=\frac{2 \pi^{2} \rho \lambda a^{2}}{\tau^{2}}
$$


where $\rho$ is density of the medium, $\lambda$ is wave-length, $\tau$ is wave period, and $a$ is amplitude. All of our observations are in the period range of 1 to 2 sec so that, for our purposes, amplitude is directly proportional to the square root of energy. Thus, from (5), (6), and (7), we can form amplitude functions for $P^{\prime}$ and $P^{\prime} P^{\prime}$

$$
\begin{aligned}
& a_{1}(\Delta)=B F_{1}(\Delta)(\sin \Delta)^{-1 / 2} \\
& a_{11}\left(\Delta^{\prime}\right)=B F_{1}(\Delta)[2 \sin (2 \Delta)]^{-1 / 2}
\end{aligned}
$$

where $B$ is a new constant, and $F_{1}(\Delta)$ is a ray parameter containing emergence angle and

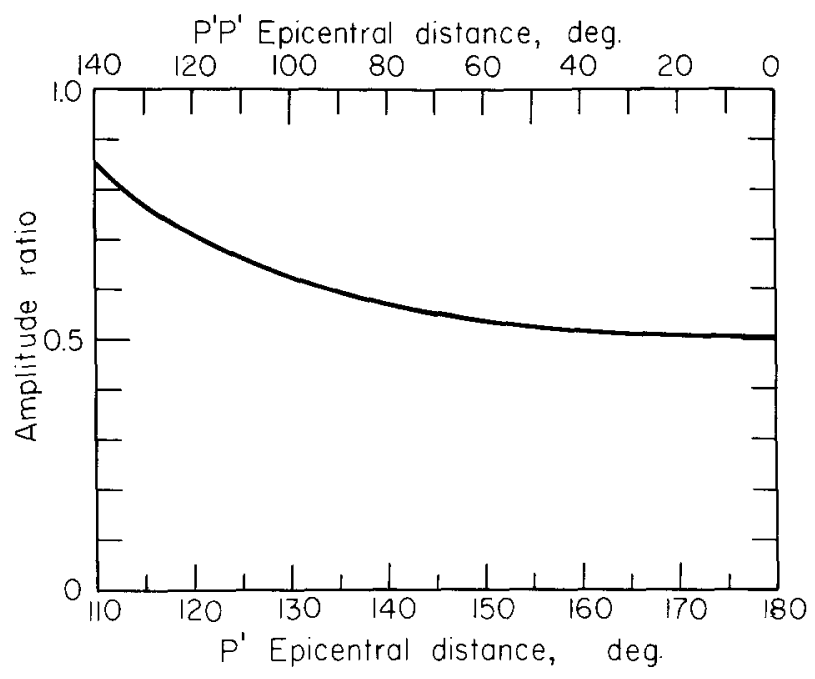

Fig. 4. Amplitude ratio of $P^{\prime} P^{\prime} \mid P^{\prime}$ from geometrical spreading.

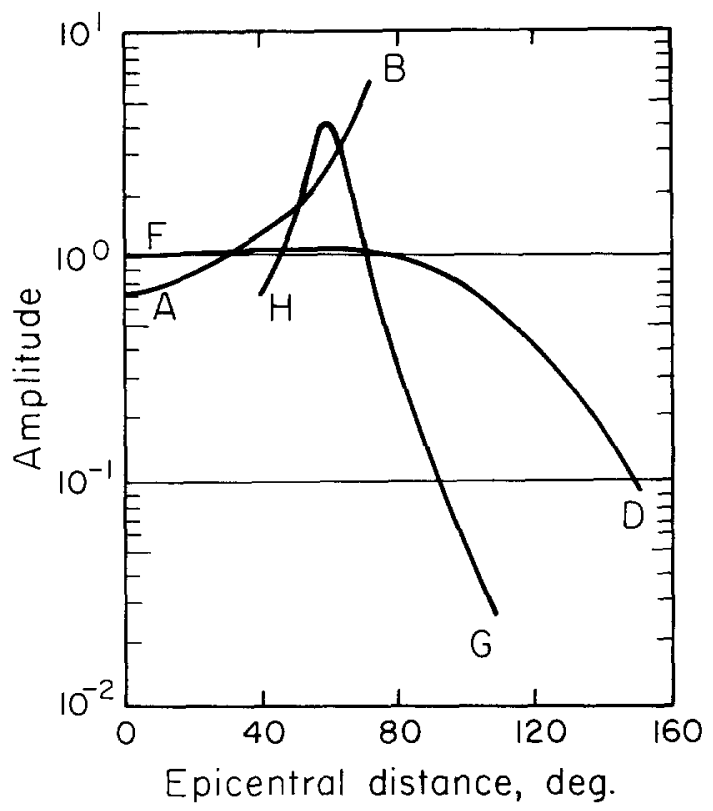

FIG. 5. Amplitudes of the branches of symmetric $P^{\prime} P^{\prime}$ calculated from observed $P^{\prime}$ amplitudes with the assumption of geometrical spreading only. Amplitudes are normalized by the amplitude of $D F$ at $0^{\circ}$ epicentral distance. 
some spreading information. Now we can see that the ratio of the symmetric $P^{\prime} P^{\prime}$ amplitude to the corresponding $P^{\prime}$ amplitude from (8) and (9) is

$$
\frac{a_{11}\left(\Delta^{\prime}\right)}{a_{1}(\Delta)}=\left[\frac{\sin (\Delta)}{2 \sin (2 \Delta)}\right]^{1 / 2}=[4 \cos \Delta]^{-1 / 2} .
$$

This function is shown in Figure 4.

We can now transform the amplitude data for $P^{\prime}$ in Figure 3 to amplitudes of $P^{\prime} P^{\prime}$ using equation (10), which involves only geometrical spreading. Figure 5 shows the $P^{\prime} P^{\prime}$ amplitudes from this calculation with the amplitudes normalized by that of the $D F$ branch at $0^{\circ}$ epicentral distance.

\section{Estimated Amplitudes of Asymmetric $P^{\prime} P^{\prime}$ From Extensive DipPing Reflectors}

In this section, we assume that the reflecting surface is large enough so that a small change in its size does not change the reflection amplitude. The geometric spreading of an asymmetric $P^{\prime} P^{\prime}$ phase is a function of the particular $P^{\prime}$ branch traveled on each leg and is also a strong function of the reflecting surface shape, which may focus or defocus the energy. The latter phenomenon is not easily resolvable and, for now, we assume that neither focusing nor defocusing takes place at the reflecting point. Spreading which is attributable to the particular $P^{\prime}$ branch is easily handled because the equivalent distance for each leg of an asymmetric $P^{\prime} P^{\prime}$ phase can be calculated and the corresponding rays and relative amplitudes for each of those distances are known. Each $P^{\prime}$ leg traveled can be used to estimate the asymmetric $P^{\prime} P^{\prime}$ amplitude. But because we are trying to set an upper limit on the amplitudes, we will simply use the largest-amplitude $P^{\prime}$ leg for our calculations unless one of the legs falls below 1 per cent of the largest-amplitude symmetric phase. Use of the largest-amplitude $P^{\prime}$ leg will compensate somewhat for any focusing effects.

The $P^{\prime} d P^{\prime}$ studies have been done in the epicentral distance range of $55^{\circ}$ to $80^{\circ}$ with the largest-amplitude branches being $G H$ between $55^{\circ}$ and $62.5^{\circ}, A B$ between $62.5^{\circ}$ and $73^{\circ}$, and $D F$ between $73^{\circ}$ and $80^{\circ}$ (Whitcomb, 1971). We want to calculate for each of these distance ranges the earliest precursor time for an asymmetric $P^{\prime} P^{\prime}$ reflection with an amplitude that is 10 per cent of the largest-amplitude phase. For time calculations, the Adams and Randall (1964) tables were used for the $G H$ branch, and the Bolt (1968) tables were used for the $A B$ and $D F$ branches. These times were shown by Whitcomb (1971) to be the best fits to the $P^{\prime} P^{\prime}$ data in the $55^{\circ}$ to $75^{\circ}$ distance range.

First we direct our attention to reflections in the great-circle plane as shown in Figure 1. Table 1 shows the asymmetric phases that give the earliest 10 per cent amplitude arrivals. The lead time shown is calculated from the time of the largest-amplitude symmetric $P^{\prime} P^{\prime}$ branch at that distance. The dip of the reflector for all of the reflections in the great-circle plane is less than $2^{\circ}$. The starred lead times are the earliest in the $G H$ and $A B$ range, but their calculated amplitudes are never significantly greater than the 10 per cent cutoff and they are neglected. Thus, the significant asymmetric $P^{\prime} P^{\prime}$ phases in the great-circle plane lead the largest-amplitude symmetric phases by times less than $18 \mathrm{sec}$.

Next, we calculate the times of asymmetric $P^{\prime} P^{\prime}$ out of the great-circle plane reflecting on a line that is perpendicular to the great circle and that is intersecting the symmetrical reflection point as seen in Figure 1. Table 1 shows that the asymmetric phases with each leg composed of the same branch are the earliest: up to $64 \mathrm{sec}$ in the $G H$ range, $35 \mathrm{sec}$ in the $A B$ range, and $83 \mathrm{sec}$ in the $D F$ range. The reflector dips range from $3^{\circ}$ to $6.6^{\circ}$. The $D F$ branch is left out of the $G H$ and $A B$ range calculations because of the same amplitude 
arguments put forth in the preceding paragraph. Because the asymmetric $P^{\prime} P^{\prime}$ out of the great-circle plane can be constructed of nearly the same rays as the symmetric $P^{\prime} P^{\prime}$, the asymmetric amplitudes are relatively large. Thus, the asymmetric $P^{\prime} P^{\prime}$ out of the greatcircle plane is the most likely to be seen as a precursor to the main phase at lead times greater than a few sec.

TABLE 1

Earliest Asymmetric $P^{\prime} P^{\prime}$ Phases with 10 Per cent Amplitudes at a Single Station

\begin{tabular}{|c|c|c|c|c|c|c|}
\hline $\begin{array}{l}\text { Largest Amplitude } \\
\text { Branch of } \\
P^{\prime} P^{\prime}\end{array}$ & $\begin{array}{l}\text { Epicentral } \\
\text { Distance } \\
\text { (deg) }\end{array}$ & $\begin{array}{c}\text { 1st } P^{\prime} \text { Leg } \\
\text { Branch-s } \\
\text { (deg) }\end{array}$ & $\begin{array}{l}\text { 2nd } P^{\prime} \text { Leg } \\
\text { Branch-s } \\
\text { (deg) }\end{array}$ & $\begin{array}{l}\text { Reflector } \\
\text { Dip } \\
\text { (deg) }\end{array}$ & $\begin{array}{l}\text { Lead Time } \\
\quad(\mathrm{sec})\end{array}$ & $\begin{array}{l}\text { Azimuth } \\
\text { Deviation } \\
\text { at Station } \\
\text { (deg) }\end{array}$ \\
\hline \multicolumn{7}{|c|}{ In Great Circle Plane } \\
\hline \multirow[t]{3}{*}{$G H\left(55^{\circ}-62.5^{\circ}\right)$} & 62.5 & GH 155 & $G H 142.5$ & $<2$ & 0.7 & 0 \\
\hline & 62.5 & $D F 180$ & $D F 117.5$ & $<2$ & $36.6^{*}$ & 0 \\
\hline & 55.0 & $D F 175$ & GH 130 & $<2$ & $45.3^{*}$ & $\mathbf{0}$ \\
\hline \multirow[t]{3}{*}{$A B\left(62.5^{\circ}-73^{\circ}\right)$} & 62.5 & GH 155 & GH 142.5 & $<2$ & 9.9 & 0 \\
\hline & 62.5 & $D F 180$ & $D F 117.5$ & $<2$ & $45.8^{*}$ & $\mathbf{0}$ \\
\hline & 62.5 & $D F 167.5$ & GH 130 & $<2$ & $39.1^{*}$ & $\mathbf{0}$ \\
\hline \multirow[t]{2}{*}{$D F\left(73^{\circ}-80^{\circ}\right)$} & 75.0 & GH 155 & GH 130 & $<2$ & 8.0 & 0 \\
\hline & 75.0 & $D F 170$ & $D F 115$ & $<2$ & 18.0 & 0 \\
\hline \multicolumn{7}{|c|}{ Out of Great Circle Plane } \\
\hline \multirow[t]{2}{*}{$G H\left(55^{\circ}-62.5^{\circ}\right)$} & 54.0 & $G H 140.5$ & $G H 140.5$ & 4.8 & 64.4 & 52 \\
\hline & 54.0 & $A B 143.5$ & $A B 143.5$ & 6.6 & 50.0 & 46 \\
\hline \multirow[t]{2}{*}{$A B\left(62.5^{\circ}-73^{\circ}\right)$} & 64.0 & $G H 143$ & GH 143 & 3.3 & 33.0 & 35 \\
\hline & 64.0 & $A B 143$ & $A B 143$ & 5.2 & 35.0 & 35 \\
\hline$D F\left(73^{\circ}-80^{\circ}\right)$ & 74.0 & $D F 121$ & $D F 121$ & 3.0 & 82.8 & 63 \\
\hline
\end{tabular}

* Small amplitudes, probably insignificant.

\section{Effect of the Finite Size of the Reflecting Surface}

The previous calculations assumed an extensive, nearly plane reflecting surface. Because the boundaries of major physical property changes in the Earth are, or have been, controlled in a rough sense by equal pressure and temperature surfaces that are approximately spherically symmetric, the boundaries themselves tend to be spherically symmetric or "horizontal". Deviations from spherical symmetry are readily observed, especially at major boundaries at or near the Earth's surface: the land-surface, oceanbottom, and Moho discontinuity for example. However, because we need nearly plane surfaces with which to reflect $P^{\prime} P^{\prime}$ waves, it is important to observe that the more a typical boundary deviates from spherical symmetry, the less extensive are its nearly planar surfaces; that is, it is rougher. For example, the ocean surface is horizontal over extensive areas, whereas steep slopes of mountains have almost no planar surfaces of dimensions larger than a few kilometers. We must, therefore, estimate what surface extent is adequate for appreciable reflection of $P^{\prime} P^{\prime}$ energy. This can be approximated by 
a calculation of the Fresnel diffraction pattern of a wave that is reflected from a semiinfinite plane with a straight edge (Born and Wolf, 1965, p. 433). A wavelength of $10 \mathrm{~km}$ and an observer distance of $12,000 \mathrm{~km}$ is used. The calculated amplitude at the observer begins to be affected appreciably when the reflector edge is within $170 \mathrm{~km}$ of the optical reflection point. Thus, for reflection surfaces of radii less than $170 \mathrm{~km}$ or diameters less than $340 \mathrm{~km}$, the amplitude will begin to be reduced because of the size of the reflection surface, and reflections from surfaces significantly smaller, say $100 \mathrm{~km}$ in dimension, will probably not be seen.

The results of Table 1 show that the earliest asymmetric $P^{\prime} P^{\prime}$ phases, based on a 10 per cent amplitude cutoff, reflect from surfaces dipping from $3^{\circ}$ to $6.6^{\circ}$. It is a difficult task to find a $3^{\circ}$ dipping surface at the ocean bottom or land surface that is nearly planar over dimensions of $100 \mathrm{~km}$. Thus, the cases in Table 1 are too extreme because of the finiteness of the asymmetric $P^{\prime} P^{\prime}$ reflector, and the earliest lead times for the rays out of the great-circle plane are probably too large. If the reflector dip is set at an upper limit of $3^{\circ}$, then the phases traveling out of the great-circle plane would be limited to lead times of $30 \mathrm{sec}$ or less for the $G H$ and $A B$ branches and $80 \mathrm{sec}$ for the $D F$ branch. If a more reasonable dip limit of $2^{\circ}$ is set, then all branches would have asymmetric $P^{\prime} P^{\prime}$ lead times less than about $20 \mathrm{sec}$.

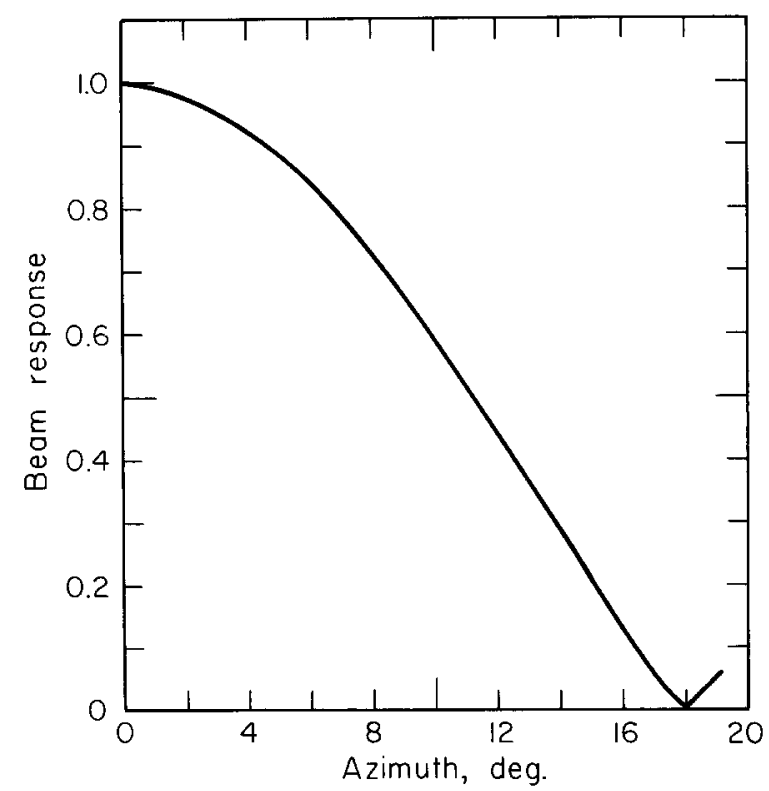

FIG. 6. LASA beam azimuth response for rings D, E, and F. A $d t / d \Delta$ of $2.0 \mathrm{sec} / \mathrm{deg}$ and frequency of $1.0 \mathrm{~Hz}$ was used.

\section{Effect of Array Processing}

Seismic arrays, through beam-forming, have the ability to band-pass filter coherent energy in both $d t / d \Delta$ and azimuth. When this is done, considerable improvement over recording at a single station can be made by elimination of the undesired asymmetric $P^{\prime} P^{\prime}$ phases. Band pass of a particular $d t / d \Delta$ eliminates all asymmetric $P^{\prime} P^{\prime}$ phases whose last $P^{\prime}$ leg has a different $d t / d \Delta$ than the largest-amplitude branch for that epicentral distance range. More importantly, band pass of arrivals from a certain azimuth eliminates those asymmetric phases traveling out of the great-circle plane which are the most bothersome type. However, because the band pass is not a delta function and has some 
width, arrivals that deviate only a little from the desired azimuth will be passed. A typical beam azimuth response is shown in Figure 6 for the LASA array in Montana for the outer three rings: $\mathrm{D}, \mathrm{E}$, and $\mathrm{F}$. A $d t / d \Delta$ of $2.0 \mathrm{sec} / \mathrm{deg}$ and frequency of $1.0 \mathrm{~Hz}$ was used. The curve shows that the amplitude response falls to 10 per cent at an azimuth of $16^{\circ}$ off the great-circle path. If this $16^{\circ}$ azimuth limit is used for the phases in Table 1, then all of the asymmetric $P^{\prime} P^{\prime}$ phases are limited to lead times less than $10 \mathrm{sec}$.

\section{CONCLUSIONS}

The possible ranges of asymmetric $P^{\prime} P^{\prime}$ reflecting at dipping interfaces at or near the Earth's surface are summarized in Figure 7. The ranges are relative to the largestamplitude symmetric $P^{\prime} P^{\prime}$ branches that are used for the study of $P^{\prime} d P^{\prime}$ phases. Three

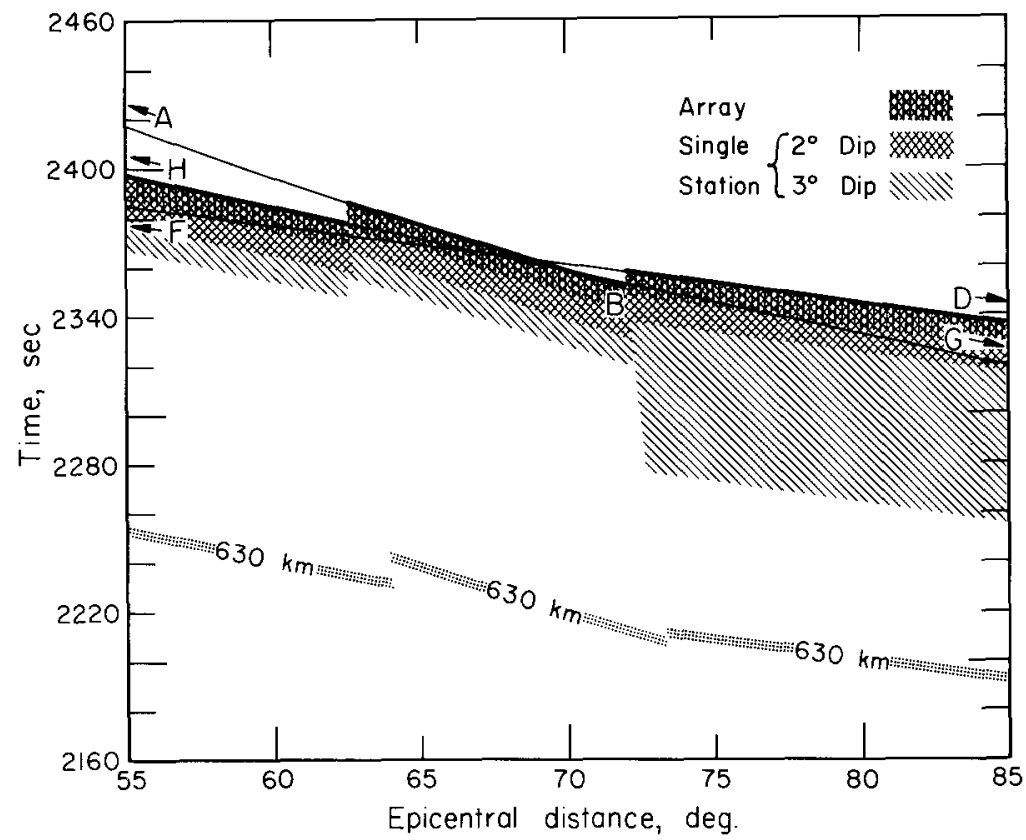

Fig. 7. A schematic summary of possible asymmetric $P^{\prime} P^{\prime}$-phase lead times recorded at a single station with $2^{\circ}$ and $3^{\circ}$ reflector dips and at a LASA-type array. The largest-amplitude branches are indicated by heavy lines and the time of a $P^{\prime} 630 P^{\prime}$ phase is shown for reference.

ranges corresponding to recording at a single station with $2^{\circ}$ and $3^{\circ}$ reflector dips and recording at a LASA-type array are shown. The calculations have been considerably simplified on the conservative side and should represent lead-time maximums that include consideration of the relative amplitudes of $P^{\prime}$ phases, the finiteness of the reflecting surfaces of dipping interfaces, and the beam-forming capabilities of a seismic array.

From Figure 7, we conclude that for lead times much less than $30 \mathrm{sec}$, equivalent to a $P^{\prime} d P^{\prime}$ with $d$ at about $110 \mathrm{~km}$, a single station cannot easily discriminate between asymmetric $P^{\prime} P^{\prime}$ and $P^{\prime} d P^{\prime}$. The $D F$ range between $75^{\circ}$ and $85^{\circ}$ may be worse, depending on the reflector dip. However, use of a beam-forming array such as LASA in Montana reduces the possible asymmetric $P^{\prime} P^{\prime}$ lead times to less than $10 \mathrm{sec}$.

Examination of $P^{\prime} P^{\prime}$ precursors at single stations in both the seismic recordings and data summaries in Whitcomb and Anderson (1970), and Whitcomb (1971) reveals that there is a significant build-up of arrivals starting at around $30 \mathrm{sec}$ lead time. A sample of 
the array data from the latter paper shown in Figure 8 also shows that much of this energy within a 30 -sec lead time is arriving at $d t / d \Delta$ 's different from those of the largestamplitude branch and other symmetric branches. This would imply that asymmetric $P^{\prime} P^{\prime}$ is indeed present. However, there are arrivals in the same figure with lead times between 10 and $30 \mathrm{sec}$ recorded at LASA with the proper $d t / d \Delta$ that indicate the presence of $P^{\prime} d P^{\prime}$ phases. Thus, we must conclude that both asymmetric $P^{\prime} P^{\prime}$ and $P^{\prime} d P^{\prime}$ are seen on the seismic record with lead times of from 0 to $30 \mathrm{sec}$. The interpretation of these phases as solely $P^{\prime} d P^{\prime}$ by studies such as Gutenberg (1960), Adams (1968), Engdahl and Flinn (1969a), Whitcomb (1971), and Adams (1971) is, therefore, in question because single stations were used for interpretation. Precursors with 0- to 30-sec lead times should be studied using seismic arrays.

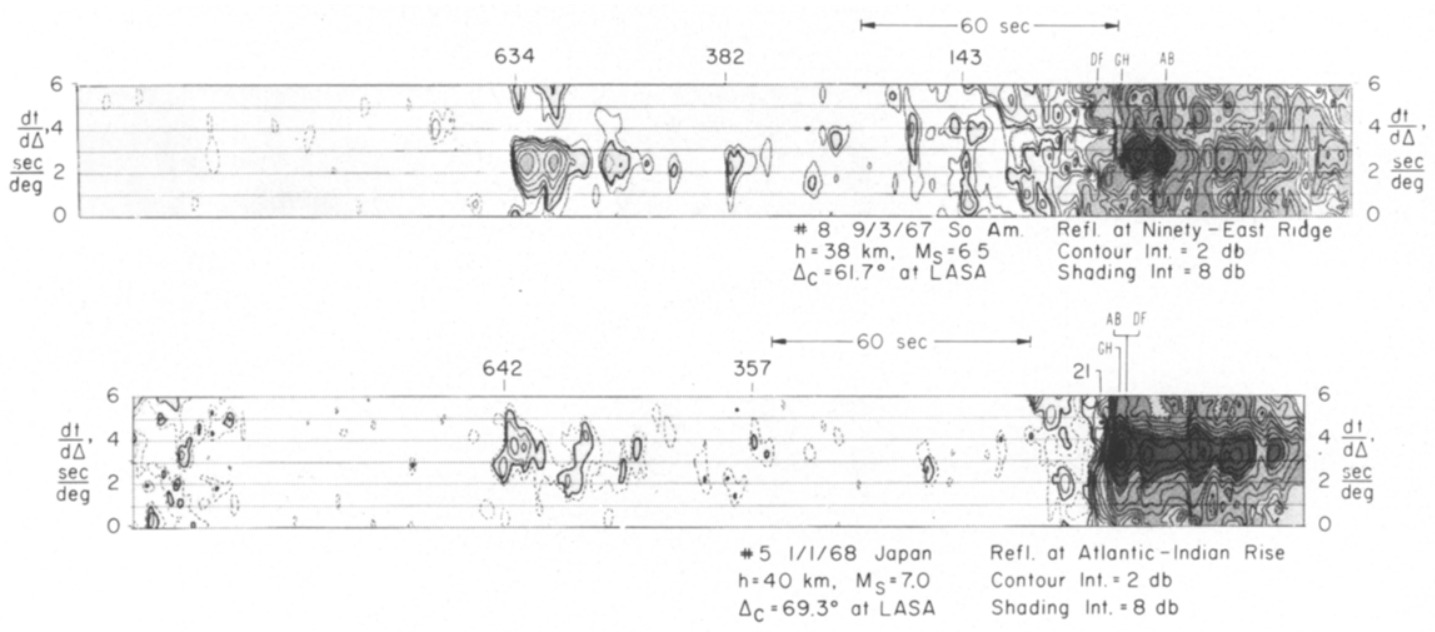

FIG. 8. Array data for two events recorded at LASA in Montana. The diagrams are contour plots of array beam power in decibels as a function of time and $d t / d \Delta$. Depths of reflection $d(\mathrm{~km})$ of $P^{\prime} d P^{\prime}$ and theoretical main branch times are shown.

Deeper reflections, such as the strong $P^{\prime} 640 P^{\prime}$ phase, can also show leading, emergent arrivals of asymmetric phases if the reflection is strong enough. This is indeed seen on good single-station recordings of the deep reflector such as the excellent example in Adams (1971, Figure 6), but the emergent arrivals are not seen in array-formed beams such as those of Figure 8.

A further effect that is apparent from the analysis of asymmetric $P^{\prime} P^{\prime}$ is that the geographic reflection point of a $P^{\prime} P^{\prime}$ phase is poorly determined. An example of this is seen in the first entry in Table 1 . An asymmetric surface reflection $6^{\circ}$ away from the symmetric reflection point differs in time from the symmetric reflection by only $0.7 \mathrm{sec}$. This is an extreme case in which the amplitude of the asymmetric phase is small, but, unless the orientation of the reflector is known, it is apparent that one cannot determine its horizontal location to within a few degrees.

\section{ACKNOWLEDGMENTS}

I am grateful to Paul G. Richards for valuable discussions and to Don L. Anderson for a critica reading of the manuscript. This work was partially supported by the Advanced Research Projects Agency of the Department of Defense and was monitored by the Air Force Office of Scientific Research under Contracts F44620-69-C-0067 and F44620-72-C-0078. During the course of the work, the author was supported by the California Institute of Technology Gutenberg Fellowship. 


\section{REFERENCES}

Adams, R. D. (1968). Early reflections of $P^{\prime} P^{\prime}$ as an indication of upper mantle structure, Bull. Seism. Soc. Am. 58, 1933.

Adams, R. D. (1971). Reflections from discontinuities beneath Antarctica, Bull. Seism. Soc. Am. 61, 1441.

Adams, R. D. and M. J. Randall. (1964). The fine structure of the Earth's core, Bull. Seism. Soc. Am. $54,1299$.

Bolt, B. A. (1968). Estimation of PKP travel times, Bull. Seism. Soc. Am. 58, 1305.

Born, M. and E. Wolf. (1965). Principles of Optics, 3rd ed., Pergamon, New York.

Buchbinder, G. G. R. (1971). A velocity structure of the Earth's core, Bull. Seism. Soc. Am. 61, 429.

Bullen, K. E. (1963). An Introduction to the Theory of Seismology, 3rd ed., Cambridge Univ. Press, Cambridge.

Engdahl, E. R. and E. A. Flinn. (1969a). Seismic waves reflected from discontinuities within the upper mantle, Science 163, 177.

Engdahl, E. R. and E. A. Flinn. (1969b). Remarks on the paper "Early reflections of $P^{\prime} P^{\prime}$ ' as an indication of upper-mantle structure" by R. D. Adams, Bull. Seism. Soc. Am. 59, 1415.

Gutenberg, B. (1960). Waves reflected at the "surface" of the Earth: $P^{\prime} P^{\prime} P^{\prime} P^{\prime}$, Bull. Seism. Soc. Am. 50, 71.

Jeffreys, H. and E. R. Lapwood. (1957). The reflexion of a pulse within a sphere, Proc. Roy. Soc. London, Series $A, \mathbf{2 4 1}, 455$.

Julian, B. R. (1970). Ray tracing in arbitrarily heterogeneous media, Mass. Inst. of Tech., Lincoln Lab., Technical Note 1970-45, 1.

Richards, P. G. (1972). Seismic waves reflected from velocity gradient anomalies within the Earth's upper mantle (unpublished data).

Shahidi, M. (1968). Variation of amplitude of PKP across the caustic, Phys. Earth Planet. Interiors 1, 97.

Whitcomb, J. H. (1971). Reflections of $P^{\prime} P^{\prime}$ seismic waves from 0 to $150 \mathrm{~km}$ depth under the Ninety-East Ridge, Indian Ocean, and the Atlantic-Indian rise, Am. Geophys. Union Monograph 14, 211.

Whitcomb, J. H. and D. L. Anderson. (1970). Reflection of $P^{\prime} P^{\prime}$ seismic waves from discontinuities in the mantle. J. Geophys. Res. 75, 5713.

Wright, C. (1972). Array studies of seismic waves arriving between $P$ and $P P$ in the distance range $90^{\circ}$ to $115^{\circ}$, Bull. Seism. Soc. Am. 62, 385.

SeIsMological Laboratory

Division of Geological and Planetary Sciences

California Institute of Technology

Pasadena, California 91109

CONTRibution No. 2179

Manuscript received May 15, 1972. 